\title{
Course variability of the atlantic (V3) segment of vertebral artery: anatomical study with clinical implications
}

\author{
M.K. Ciołkowski1, 2(1), B. Ciszek ${ }^{1,3}$ (1) \\ ${ }^{1}$ Department of Descriptive and Clinical Anatomy, Centre of Biostructure Research, Medical University of Warsaw, Poland \\ 2Department of Neurosurgery, Children's Memorial Health Institute, Warsaw, Poland \\ ${ }^{3}$ Department of Neurosurgery, Prof. Bogdanowicz Children Hospital, Warsaw, Poland
}

[Received: 16 November 2019; Accepted: 27 January 2020]

\begin{abstract}
Background: The atlantic segment of vertebral artery (V3) located at the centre of the cranio-vertebral junction is known for its variability and asymmetry, and is either the target or on the way of numerous procedures in this region. The aim of the study was to visualise variability of $\mathrm{V} 3$ segment.

Materials and methods: The V3 segment was studied in 49 specimens of the suboccipital region injected with coloured gelatine. Direct measurements were performed and probabilistic maps were created using digital photography.

Results: The V3 segment has wavy course with possible lateral and significant postero-inferior expansions. In relation to the foramen transversarium the $V 3$ reached up to $5 \mathrm{~mm}$ laterally, $23 \mathrm{~mm}$ posteriorly, $27 \mathrm{~mm}$ medially and up to $11 \mathrm{~mm}$ downward. Looking from the medial aspect the course of the V 3 is less predictable compared to the lateral approach. Linear measurements and probabilistic maps revealed significant variability and large range of variation. There were 11 cases of V3 tortuosity found in studied material.

Conclusions: The complex and variable spatial conformation of V3 makes individual diagnostic and preoperative approach necessary. (Folia Morphol 2021; 80, 1: 20-25)
\end{abstract}

Key words: atlanto-occipital joint, cranio-cervical junction, suboccipital triangle, cranio-vertebral junction, vertebral artery anatomy, vertebro-basilar system

\section{INTRODUCTION}

Last decades brought significant advance in diagnosis and treatment of the cranio-vertebral junction (CVJ) deformities and injuries. The dedicated instrumentation and surgical techniques have been developed to accommodate specific structure and function of this region. Very close and complex relationships of the vertebral artery (VA) and the CVJ structures make an individual approach with precise vascular imaging a gold standard and necessity in many cas- es. Nevertheless, awareness of anatomical variability range and spectrum of possible anomalies is essential for surgeon planning and analysing the diagnostic imaging, as well as in any emergency or unexpected situations. The midline approaches to the posterior cranial fossa, foramen magnum and upper part of the cervical vertebral canal are naturally limited on both sides by the VAs piercing the dura and reaching cranial cavity. Moreover the lateral and far-lateral posterior fossa approaches have the VA in the centre

Address for correspondence: M.K. Ciołkowski, MD, PhD, Department of Descriptive and Clinical Anatomy, Centre of Biostructure Research, Medical University of Warsaw, ul. Chałubińskiego 5, 02-004 Warszawa, Poland, tel/fax: +48 2262952 83, e-mail: maciej.ciolkowski@wum.edu.pl 
of operative field and frequently involve its dissection or transposition. The arch of VA surrounding the atlanto-occipital joint may be in conflict with spine instrumentation in degenerative or traumatic cases. The aim of this study was to estimate the variability of the atlantic segment of the VA as it is a crucial anatomical structure during procedures around CVJ.

The VA is divided into four segments according to their topography and development: V1 — prevertebral, V2 - cervical (also referred to as intertransversary), V3 - atlantic, and V4 - intracranial (actually — intradural) [8]. Despite some ambiguity in understanding borders of these segments by different authors, the atlantic segment is defined here as the one extending between foramen transversarium of atlas vertebra and dura crossing $[10,17]$. This assumption is justified on embryological and topographical grounds.

\section{MATERIALS AND METHODS}

Fifty specimens of the posterior CVJ structures and suboccipital soft tissues harvested during routine forensic autopsies were studied in accordance with local ethico-legal regulations. The studied individuals had no medical records or signs on gross examination of direct trauma or surgical interventions within CVJ. There were no signs of significant atherosclerosis or stenosis of the studies arteries. A case of atlas assimilation was excluded from this cohort and described elsewhere [4], while another case of VA duplication around the posterior arch of atlas [5] was left within the analysed group. Cadavers examined here included 34 males and 15 females, aged 18 to 91 years (mean 53 years, standard deviation 18.25).

The arteries were injected with coloured gelatine and specimens were fixed in formalin. After gross dissection of the nuchal muscles to the level of suboccipital musculature a microsurgical dissection was performed to visualise whole length of the V3 bilaterally - total 98 segments of VA were studied.

All linear measurements of the specimens were taken directly using calliper of $0.02 \mathrm{~mm}$ accuracy and basic statistical analysis was performed with Statistica 9.0. Preparations were immobilised with the triangle determined by the tips of the transverse processes and the posterior tubercle set in the horizontal plane. Digital pictures were taken of each specimen in three cardinal planes (superior, posterior and lateral views) with reference ruler. Further analysis was performed separately for each side to avoid influence of variability in the general width of the atlas vertebra. Based on the calibrated images the simplified models of VA projections were created using the Bézier Curves tool in CorelDraw ${ }^{\circledR}$ X3 graphic platform. If more than four 'nodes' of the Bézier Curves tool were needed to model the superior V3 projections due to sudden turn or inflection of the artery curvature, such vessel was regarded as tortuous. The vector graphics models were superimposed on each other with different landmarks used as a common point. Using the transparency tool in the multi-layered picture, the probabilistic maps of VA position were created (each projection model of all 49 was given transparency level of $98 \%$ ) and presented on $1 \mathrm{~mm}$ grid.

\section{RESULTS}

The VA soon after leaving the foramen transversarium bent posteriorly and downwards above the posterior bar of the transverse process in all studied specimens and started a course curved in all three planes. In some cases the tip of the VA arch 'hanged' down as far as $11 \mathrm{~mm}$ below the upper surface of the transverse process as observed from the side (Fig. 1). Thus the proximal arm of the V3 run downward and the distal one - upward to reach the groove on the posterior arch of atlas.

Observation of the superior views revealed three general types of initial VA course after leaving the foramen transversarium of atlas. Rarely the artery directed immediately towards midline (type A - 4.1\% on the left and $14.3 \%$ on the right). Sometimes it first took sagittal course defined arbitrary as fitting the $10^{\circ}$ parasagittal angle (type $B-10.2 \%$ on the left and $28.6 \%$ on the right). Most often it run slightly lateral (type C $-85.7 \%$ on the left and $57.1 \%$ on the right) to form wider arch surrounding the atlanto-occipital joint (Fig. 2).

The superior projections were analysed from two points of view. Taking the foramen transversarium and the beginning of the V3 segment as the common point resulted in creation of maps simulating location of the VA as if dissected from the side of the transverse process (Fig. 3). The arch of V3 seems to be predictable and conformed to the geometry of the superior articular surface and the whole joint when seen on these pictures. Relatively large 'danger zone' compared to the probabilistic map should also be noticed - it is caused by cases of tortuous V3 separately presented further. It extends from the common point laterally up to $5 \mathrm{~mm}$, posteriorly up to $23 \mathrm{~mm}$ and medially up to $27 \mathrm{~mm}$. In order to simulate dissection 


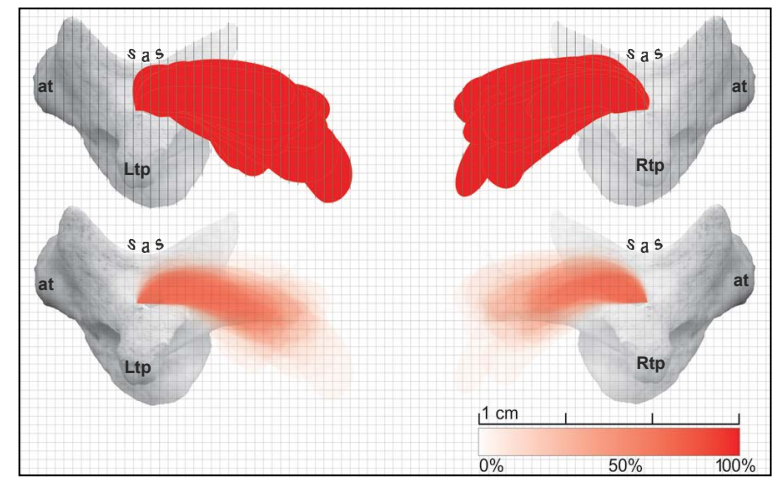

Figure 1. Cumulated lateral views of the left and right V3: marking the 'danger zone' above and the probabilistic map below; at anterior tubercle of atlas; Ltp, Rtp — left and right transverse process; sas - superior articular surface of atlas.

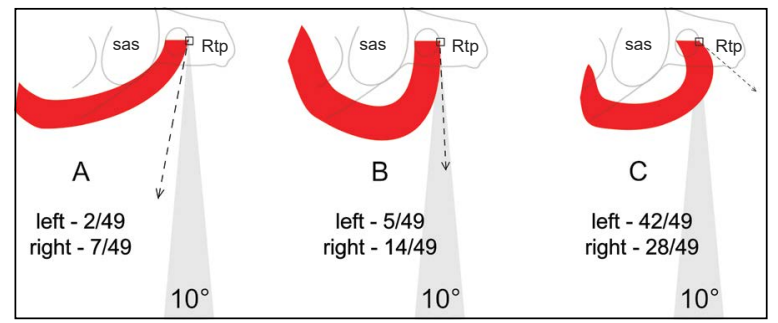

Figure 2. Three possible general directions of the V3 course after leaving the foramen transversarium (type A - medial, B - sagittal, and C - lateral) presented on selected cases; Rtp — right transverse process; sas - superior articular surface of atlas.

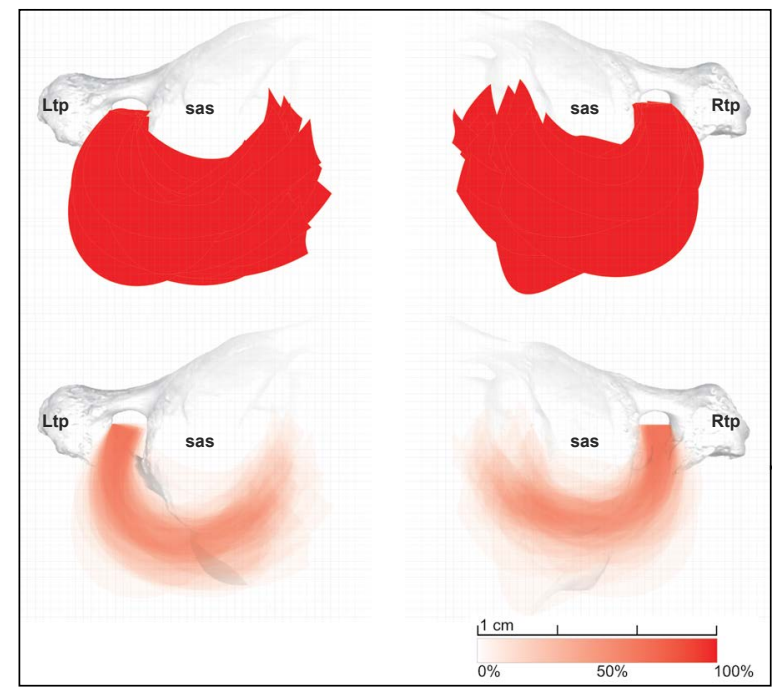

Figure 3. Superior views of the left and right V3 with the common origin in the foramen transversarium: the 'danger zone' above and the probabilistic map below; Ltp, Rtp — left and right transverse process; sas - superior articular surface of atlas.

from the midline, other maps were created taking as the common point the crossing of the inner border

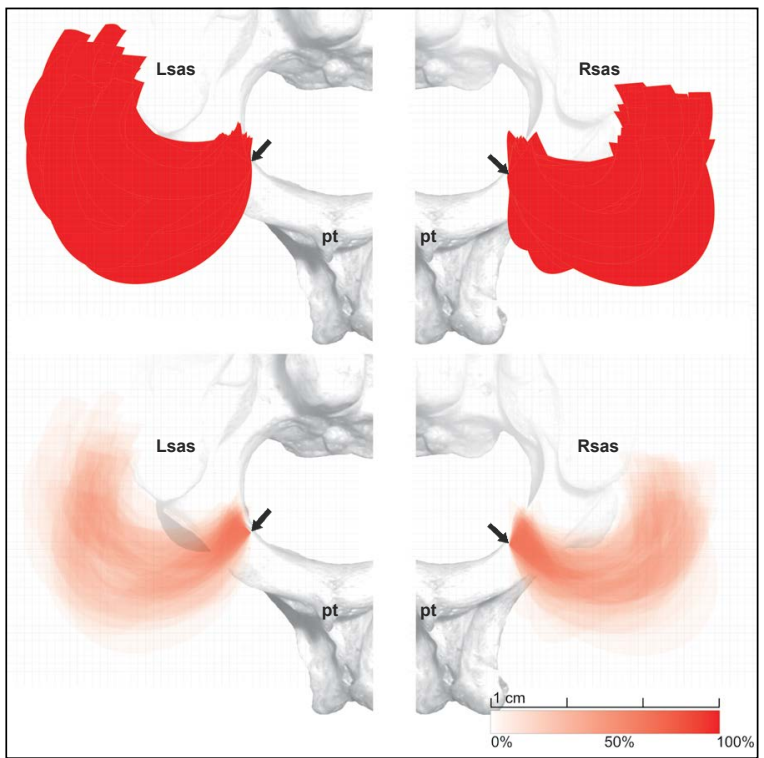

Figure 4. Superior views of the left and right V3 with the common point set close to the dura crossing (arrow): the 'danger zone' above and the probabilistic map below; Lsas, Rsas — left and right superior articular surface of atlas; $\mathrm{pt}$ - posterior tubercle of atlas.

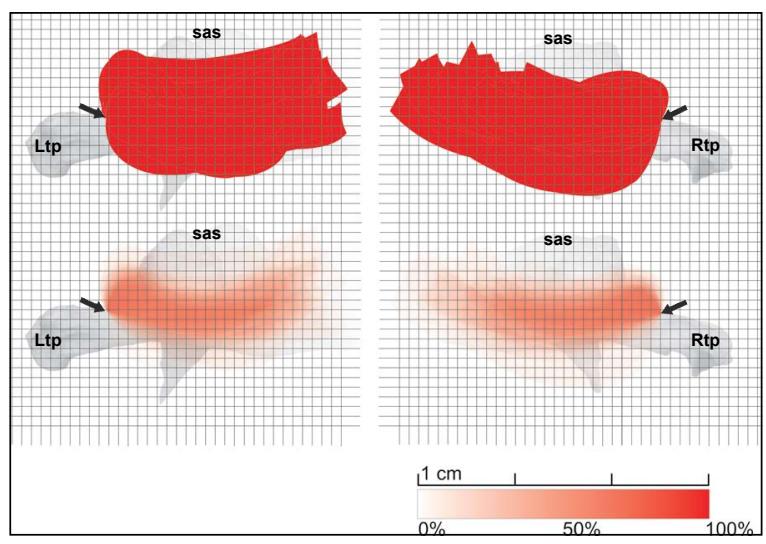

Figure 5. Posterior views of the left and right V3 with the common point in the foramen transversarium (arrow): the 'danger zone' above and the probabilistic map below; Ltp, Rtp — left and right transverse process; sas - superior articular surface of atlas.

of the posterior atlantic arch with the outer margin of the VA arch (Fig. 4). These pictures strikingly differ from the previous ones. Except for the part of the V3 located in its bony groove on the posterior arch of atlas the chance of conflict with the VA is evenly distributed in the large area extending up to $30 \mathrm{~mm}$ laterally and $16 \mathrm{~mm}$ posteriorly.

Analysis of the posterior views of the V3 (Fig. 5) together with the lateral views gives general picture of the waveform course of this artery around the atlanto-occipital joint. The posterior views were also 


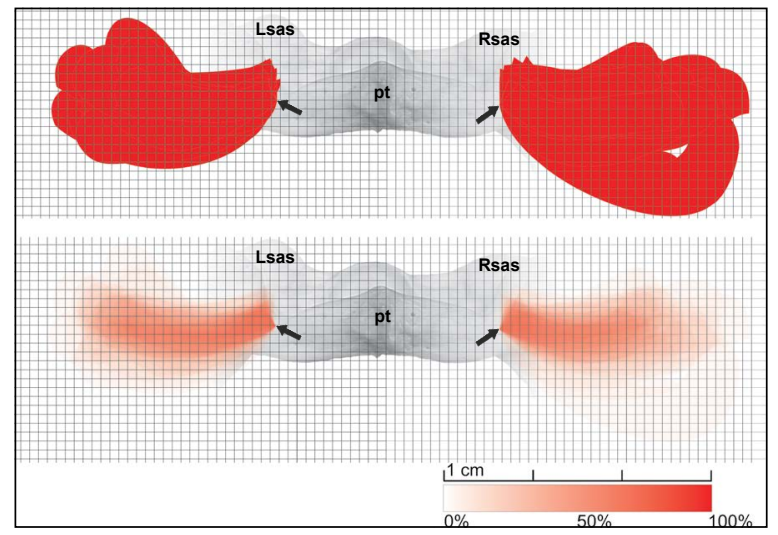

Figure 6. Posterior views of the left and right V3 with the common point at the medial (distal) end (arrow): the 'danger zone' above and the probabilistic map below; Lsas, Rsas - left and right superior articular surface; $\mathrm{pt}$ - posterior tubercle of atlas.

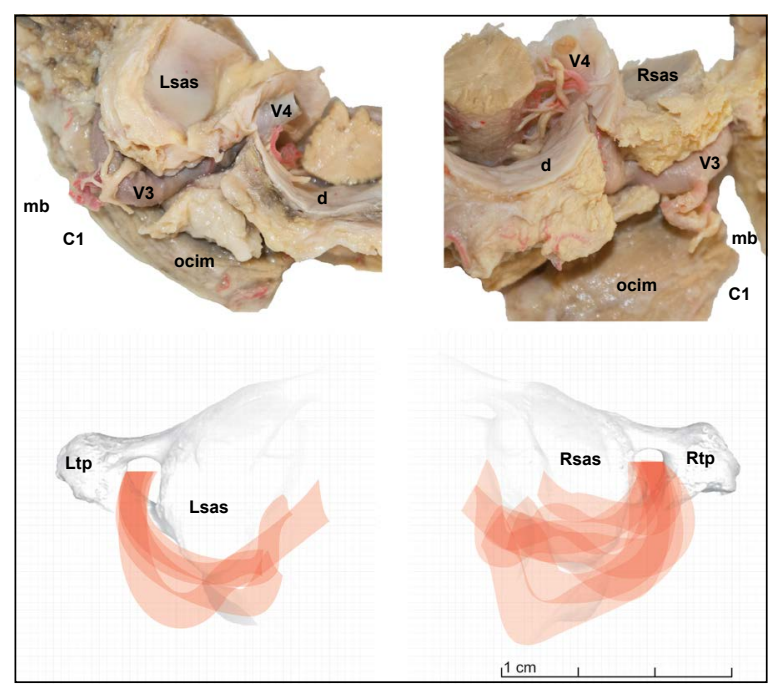

Figure 7. Above - left and right exemplary cases of tortuous V3. Pictures taken from oblique perspective. Below - superimposed superior views of all tortuous cases; Lsas, Rsas — left and right superior articular surface of atlas; $d$ - dura mater cut right below foramen magnum; ocim — obliquus inferior capitis muscle; V3, V4 - atlantic and intradural segments of vertebral artery; $\mathrm{mb}$ muscular branch of $\mathrm{V} 3 ; \mathrm{C} 1$ - dorsal ramus of spinal nerve $\mathrm{C}$; Ltp, Rtp — left and right transverse process of atlas.

studied taking the distal end of the arteries as the common point with the conclusions similar to these obtained from the horizontal projections (Fig. 6).

In the great majority of cases it was enough to use four 'nodes' of the Bézier Curves tool to model the superior projections of the VA. However in some cases $(11.2 \%)$ additional nodes were necessary to model the more complex V3 curvature. These cases were referred to as tortuous: 4 on the left and 7 on the right side (Fig. 7). Abnormalities in the course of

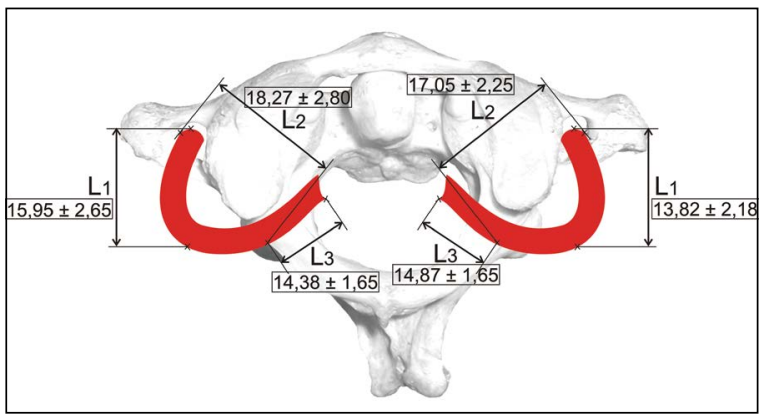

Figure 8. The mean values and standard deviations of direct linear measurements of the V3 (L1-L3) - details in text.

the $\mathrm{V} 3$ concentrate along its distal part. The tortuous vertebral arteries were found in rather shallow bony grooves of the atlas.

The length of the proximal part of the V3 arch (L1) was measured from the transversary foramen to the tip of the arch - usually located in the axis of the atlanto-occipital joint (Fig. 8). The chord of the V3 arch (L2) was measured between the foramen transversarium and the crossing of the artery with the posterior arch of atlas. The index L1/L2 represents general shape of the arch - the bigger value, the arch is "slimmer". The sagittal dimension of the left V3 arches is significantly longer compared to the right arteries, and the left arches are more "slim" (Table 1). The length of the artery in the bony groove until the dura crossing (L3) was comparable on both sides.

\section{DISCUSSION}

Lang and Kessler [12] described the dorsal course of the VA after leaving the foramen transversarium of atlas vertebra as a rule. Ulm et al. [15] basing on dissections and angiographic studies described course of the VA after leaving the foramen transversarium of atlas as medial. The studies of Abd El-Bary et al. [1] and Cacciola et al. [3] revealed sagittal or medial course of the VA. These discrepancies may originate from subjective observations of the course of the VA, which are difficult because of waveform shape of the artery and oblique axis of the atlanto-occipital joint. On the other hand Yamaguchi et al. [16] in study based on the large radiological material pointed the postero-lateral protrusion of the VA over the posterior arch of atlas. In the material studied for this paper we found wide range of shapes of the $\mathrm{V} 3$ arch, where wide arches with initially postero-lateral course prevail. As the arteries dissected in our study were relatively stiff after prior injection in situ, fixation and 
Table 1. Statistical analysis of the V3 linear measurements in millimetres

\begin{tabular}{|c|c|c|c|c|c|c|c|}
\hline & \multicolumn{3}{|c|}{ Left } & \multicolumn{3}{|c|}{ Right } & \multirow[t]{2}{*}{$\mathbf{P}$} \\
\hline & Range & Mean & $\pm S D$ & Range & Mean & \pm SD & \\
\hline L1 & $9.86-23.22$ & 15.95 & 2.65 & $9.70-20.70$ & 13.82 & 2.18 & 0.000039 \\
\hline L2 & $12.08-27.84$ & 18.27 & 2.80 & $13.24-24.60$ & 17.05 & 2.25 & 0.020087 \\
\hline L1/L2 & $0.55-1.43$ & 0.88 & 0.16 & $0.61-1.18$ & 0.82 & 0.11 & 0.016084 \\
\hline L3 & $11.10-18.92$ & 14.38 & 1.65 & $10.72-18.46$ & 14.87 & 1.65 & 0.139438 \\
\hline
\end{tabular}

L1 — length of the V3 arch, L2 - width of the V3 arch, L3 - length of the V3 in the bony groove. SD — standard deviation, p-value for t-Student test of significant difference between sides

preservation of anchoring bone or dura, observations and measurements are more likely to represent reality.

The asymmetry of the initial course of VA in horizontal plane with more frequent medial deviation of the right artery and lateral course in great majority of left arteries could be explained by handedness; however, there are no data to verify such hypothesis in our material. Right hand and eye domination of the investigator might have also influenced presented results, at least partially.

The authors of clinically oriented papers $[1,6,9$, $11-13,15]$ analysed statistically linear measurements taken mainly in cardinal planes or between arbitrary chosen and easily defined points, which is of limited value in the operative field and makes understanding the real position of the vessel difficult. This problem we tried to overcome with our graphic method. General assessment of our probabilistic maps indicates higher uncertainty of VA location when it is dissected from the medial side. Thus the strategy to avoid seeing 'naked' artery as long as possible is justified [17]. On the other hand, starting dissection at the transverse process one should remember about possible lateral and significant posterior expansion of the VA. Also the uncertain vertical extent of V3, both above the level of foramen transversarium and below, needs caution when approached from different directions [2].

The course of the VA may interfere with screw trajectory for occipito-cervical fusion procedures. The entry point for occipital condyle screw is located in the middle of posterior aspect of the condyle - i.e. above and anterior to the V3 arch $[7,11,13]$. Lee et al. [13] showed that in a significant number of patients there is no sufficient space between the occipital squama and V3 to perform this procedure. The so-called "slim" V3 arches may give more space for instrumentation. Any approach to the posterior aspect of the occipital condyle may be disturbed by tortuosity of the VA as well. The screw is inserted into the lateral mass of atlas in the middle of its width, right below the pedicle of posterior arch - i.e. anterior and below the V3 arch. The cases of V3 arch "hanging" down from the plane of transverse process and posterior arch would need cautious transposition.

Few researchers measured the length of the V 3 $[1,3,12]$ and due to different definitions of the segment or subsegments their results are not comparable. The range of $\mathrm{V} 3$ lengths revealed in our study makes any predictions pointless from practical point of view - planning of intravascular interventions or vascular reconstructions evidently needs individual approach. Significant subset of tortuous V3 revealed in our study may explain many unexpected intraoperative difficulties [14].

Comparison between graphic maps with extensive 'danger zones' and mean values of linear measurements, even completed with some information on distribution, shows general pitfall of using statistical information derived from anatomical studies. If complications are to be avoided, the range of deviations is to be analysed, not the statistical mean values. Three-dimensional statistical analysis of the VA has not been so far conducted to our best knowledge, and the maps presented here may serve as some simple forerunner of it. In this context, it seems the modern radiological studies allowing volume reconstructions are best suited for exact preoperative planning $[4,5,9-11]$.

\section{CONCLUSIONS}

The atlantic segment of the VA usually has waveform course from the foramen transversarium, behind the atlanto-occipital joint, toward the cranio-spinal dura. Nevertheless, possibility of tortuosity or significant deviations in shape and location of this artery make detailed, three-dimensional preoperative diag- 
nostics necessary in cases where direct exposure of the VA is expected. The abilities of navigation devices and virtual reality should be widely used both in patient management and in training to avoid complications and give sense of variability range.

\section{Acknowledgements}

The authors appreciate cooperation and support from Prof. Paweł Krajewski, head of the Forensic Medicine Department in Medical University of Warsaw.

\section{REFERENCES}

1. Abd El-Bary TH, Dujovny M, Ausman JI. Microsurgical anatomy of the atlantal part of the vertebral artery. Surg Neurol. 1995; 44(4): 392-400; discussion 400, doi: 10.1016/0090-3019(95)00033-x, indexed in Pubmed: 8553261.

2. Bruneau M, Cornelius JF, George B. Antero-lateral approach to the V3 segment of the vertebral artery. Neurosurgery. 2006; 58(1 Suppl): ONS29-35; discussion ONS29, doi: 10.1227/01.neu.0000193930.74183.42, indexed in Pubmed: 16479626.

3. Cacciola F, Phalke U, Goel A. Vertebral artery in relationship to C1-C2 vertebrae: an anatomical study. Neurol India. 2004; 52(2): 178-184, indexed in Pubmed: 15269464.

4. Ciołkowski MK, Krajewski P, Ciszek B. A case of atlas assimilation: description of bony and soft structures. Surg Radiol Anat. 2014; 36(8): 833-836, doi: 10.1007/s00276013-1235-9, indexed in Pubmed: 24240817.

5. Ciołkowski MK, Krajewski P, Ciszek B. A case of vertebral artery duplication at the level of atlas: anatomical description. Eur Spine J. 2014; 23 Suppl 2: 285-287, doi: 10.1007/ s00586-014-3304-0, indexed in Pubmed: 24748466.

6. Duan S, Lv S, Ye F, et al. Imaging anatomy and variation of vertebral artery and bone structure at craniocervical junction. Eur Spine J. 2009; 18(8): 1102-1108, doi: 10.1007/ s00586-009-0925-9, indexed in Pubmed: 19288143.

7. El-Gaidi MA, Eissa EM, El-Shaarawy EAA. Free-hand placement of occipital condyle screws: a cadaveric study. Eur Spine J. 2014; 23(10): 2182-2188, doi: 10.1007/s00586014-3488-3, indexed in Pubmed: 25070792.
8. FIPAT Terminologia Neuroanatomica. Federative International Programme for Anatomical Terminology. 2017. https://fipat.library.dal.ca/ (February 2017).

9. Ha W, Yang D, Gu S, et al. Anatomical study of suboccipital vertebral arteries and surrounding bony structures using virtual reality technology. Med Sci Monit. 2014; 20: 802-806, doi: 10.12659/MSM.890840, indexed in Pubmed: 24829084.

10. Harnsberger HR, Osborn AG, Macdonald AJ. Imaging anatomy: brain, head \& neck, spine. Amirsys Pub Inc 2006.

11. La Marca F, Zubay G, Morrison T, et al. Cadaveric study for placement of occipital condyle screws: technique and effects on surrounding anatomic structures. J Neurosurg Spine. 2008; 9(4): 347-353, doi: 10.3171/ SPI.2008.9.10.347, indexed in Pubmed: 18939920.

12. Lang J, Kessler B. About the suboccipital part of the vertebral artery and the neighboring bone-joint and nerve relationships. Skull Base Surg. 1991; 1(1): 64-72, doi: 10.1055/s-2008-1056982, indexed in Pubmed: 17170824.

13. Lee HJ, Choi DY, Shin MH, et al. Anatomical feasibility for safe occipital condyle screw fixation. Eur Spine J. 2016; 25(6): 1674-1682, doi: 10.1007/s00586-016-4399-2, indexed in Pubmed: 26831538.

14. Osorio JA, Benet $A$, Hess $C P$, et al. Primary vertebral artery reanastomosis during retrosigmoid skull base approach following iatrogenic near-transection with monopolar electrocautery. Neurosurgery. 2014; 10 Suppl 4: 631-639, doi: 10.1227/NEU.0000000000000526, indexed in Pubmed: 25181436.

15. Ulm AJ, Quiroga M, Russo A, et al. Normal anatomical variations of the $V_{3}$ segment of the vertebral artery: surgical implications. J Neurosurg Spine. 2010; 13(4): 451-460, doi: 10.3171/2010.4.SPINE09824, indexed in Pubmed: 20887142.

16. Yamaguchi S, Eguchi K, Kiura $Y$, et al. Posterolateral protrusion of the vertebral artery over the posterior arch of the atlas: quantitative anatomical study using three-dimensional computed tomography angiography. J Neurosurg Spine. 2008; 9(2): 167-174, doi: 10.3171/ SPI/2008/9/8/167, indexed in Pubmed: 18764749.

17. Youssef AS, Uribe JS, Ramos E, et al. Interfascial technique for vertebral artery exposure in the suboccipital triangle: the road map. Neurosurgery. 2010; 67(2 Suppl Operative): 355-361, doi: 10.1227/NEU.0b013e3181f741f7, indexed in Pubmed: 21099558. 